\title{
Attracting and Maintaining Demographic Diversity in Energy: Lessons from Petroleum Engineering.
}

\author{
Tatyana Plaksina, \\ Department of Chemical and Petroleum Engineering, Schulich School of Engineering, \\ University of Calgary, Canada \\ tatyana.plaksina@ucalgary.ca
}

\begin{abstract}
Diversity is important for any organization, its development and growth. However, frequently we observe that, if left to their own devices, many social and professional communities become selfselective, less susceptible to change, and, thus, less diverse. For example, this phenomenon can be readily observed in the academia when a professor forms a research group that is mostly comprised of people from certain origin or personal qualities. While on an individual level, this situation can be quite convenient and even beneficial, on the large scale the lack of diversity creates severe distortions and discourages many talented individuals from joining the group, organization, or industry in general.

Diversity that includes ethnic, age, and gender aspects is a serious concern in overall energy industry and academia. One of the most prominent field impacted by diversity problems is petroleum engineering which can be used as a learning opportunity for other branches of energy industry. Using petroleum engineering community, we can understand what processes have led it to become self-selective, what measures have been applied to changes the situation and introduce more diversity.
\end{abstract}

Keywords: petroleum engineering education; demographic statistical data; faculty distribution; diversity issues; gender and ethnic diversity

\section{Introduction}

Phillips in her Scientific American article outlined how being surrounded by people from different cultures and backgrounds promotes creativity, diligence, and hard work inside a diverse group [1]. The author emphasized that interaction between people with different focuses yields higher productivity, better problem solving, and broader perspective. Unfortunately, most energy engineering communities are still exhibiting poor diversity. In fact, most of them quickly become self-selective and discourage people with different ethnic, gender, and educational backgrounds from becoming a part of these groups. In this study, we investigate a locomotive of the 
energy field - petroleum engineering - and observe on statistical data from petroleum engineering academic programs how poor diversity and self-selection manifests itself in this most prominent energy community.

The choice to analyze data from petroleum engineering university programs in the US and Middle Eastern region is not accidental. First, unlike information about corporate workers, the data including academic rank, gender, and the country of origin are readily available on universities' official websites. Second, academic staff composition is usually representative of the actual diversity in the field as the same $\mathrm{PhD}$ graduates work in both industry and academic institutions (here we consider only positions that require $\mathrm{PhD}$ qualification, $\mathrm{MSc}$ and $\mathrm{BS}$ are not considered as there are no equivalent academic positions for holders of these degrees). Final, petroleum engineering is the biggest and most influential field in the energy technology industry, thus, all conclusions drawn from this analysis would be relevant to most of other smaller energy fields.

\section{Methodology}

In the previous section, we highlighted the importance of maintaing diversity inside then energy community for better problem solving and overall health of the industry. To assess the situation in petroleum engineering field as a flagman of the energy technology industry, we collect and analyze the statistical data from 14 US petroleum engineering university program and 4 similar programs in the Middle East. While looking at the data, we specifically focus on rank distribution of faculty members (meaning, only tenure-track professors) to establish approximate age diversity of the academic staff, faculty ethnic background (determined from the country where he or she obtained bachelor degree, as majority of people tend to go for the first degree in their home country), and finally gender diversity (that could only be established for those representatives who provided profile pictures if their name cannot be used for identification purposes). Because many Middle Eastern programs do not provide pictures of their faculty members on their offical websites, a very small sample of programs (four of them) that do provide this means of identification was analyzed here. Thus, in this study, we consider 14 US programs including Texas A\&M University, TX, University of Texas in Austin, TX, Penn State University, PA, Texas Tech University, TX, University of Houston, TX, Louisiana State University at Baton Rouge, LA, University of Louisiana at Lafayette, LA, University of Tulsa, OK, University of Oklahoma, OK, New Mexico Institute of M\&T, NM, West Virginia University, VA, University of North Dakota, ND, and University of Alaska, AK. From the Middle Eastern universities we selected four including Abu Dhabi Petroleum Institute, UAE, Texas A\&M University in Qatar, Amirkabir University, Iran and University of Tehran, Iran. 
As shown in Fig. 1, the academic staff in the US universities was conservatively growingover last 15 years even though the number of $\mathrm{PhD}$ students was rapdly doubling and tripling from the number of students in 2000. This low sensitivity of the faculty count to the increased number of students can be explained by relative unattractiveness of academic careers in petroleum engineering in comparison to those in oil and gas operating companies. While there is a great mobility of highly qualified experts from the industry to academic institutes, most people who choose to become a university professor as a first option usually make a lifetime career choice that unfortunately they cannot not change later on. There are almost no examples of a petorleum engineering university professor who would assume a position in a major corporation as a full time employee. As a result, most $\mathrm{PhD}$ graduates try to find industrial positions first and then consider academic career.

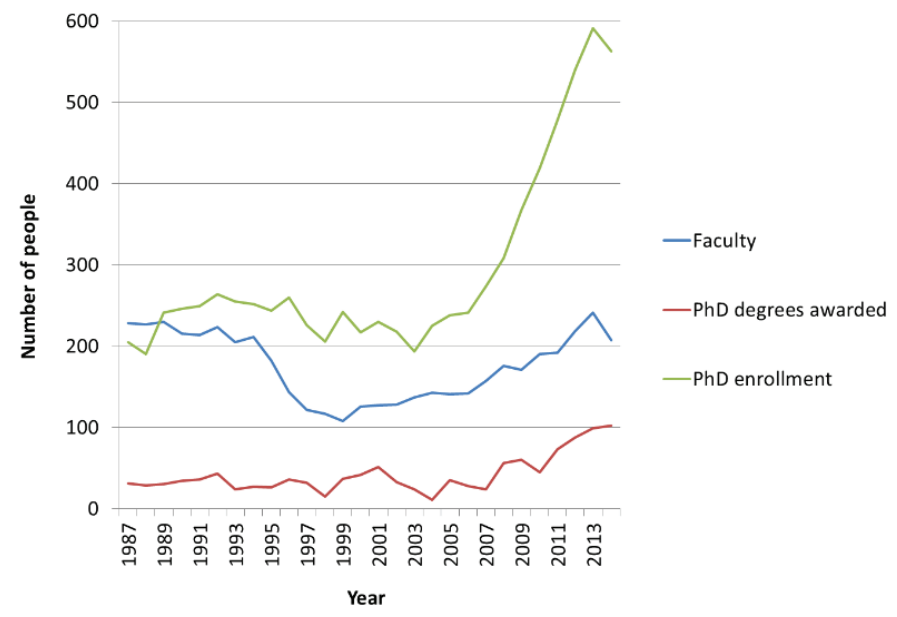

Figure 1. Faculty hiring and $\mathrm{PhD}$ enrollment and graduation trends from 1987 to 2014 in the US.

\section{Results}

Now, let us observe how these distortions affect the diversity of people in petroleum engineering faculty. Because of the low mobility between the academia and industry, people with certain backgrounds tend to join the university faculty. Fig. 2(a) demonstrates how the academic community becomes less diverse and self-selective due to certain restrictions to a certain population of $\mathrm{PhD}$ holders. Specifically, second largest percent (42 faculty members) of petroleum engineering academic staff in the US comes from the Middle East. This might look surprising because the number of PhD's from the Middle East to other nationals in petroleum engineering in the US is not that high. However, it is important to keep in mind that for a long time nationals from Iran were not eligible for any industrial positions inside the US. 
As a result, many of $\mathrm{PhD}$ graduated would gladly accept an academic position as means of obtaining legal status in the country.

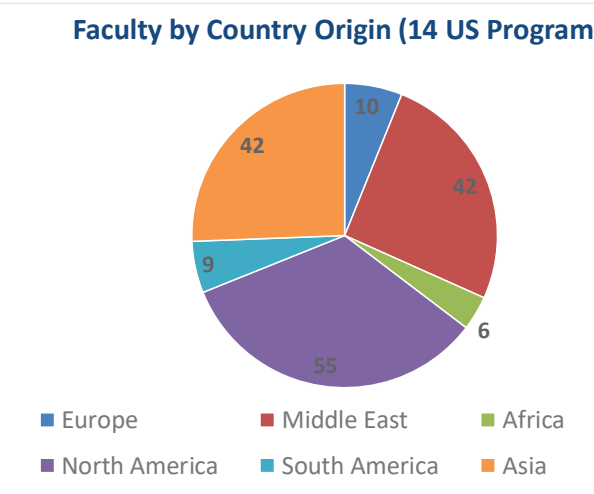

(a)

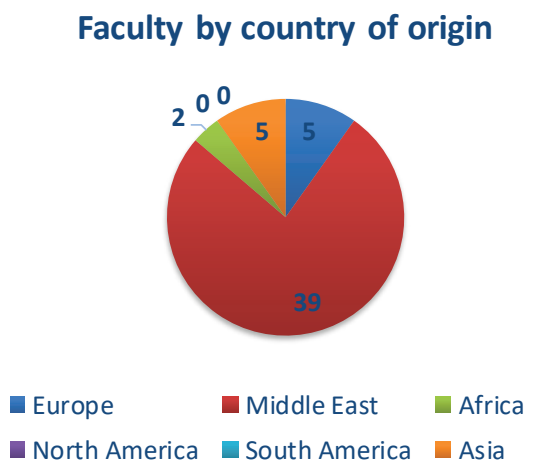

(b)

Figure 2. Faculty distibution in (a) the US and (b) in the Middle East based on their country of origin (or the country where they obtained their bachelor degree).

While this situation is not too detrimental to diversity on a scale of one school, taken to the national level, it might create tremendous problem. Specifically, professors of certain background (let us say, Iran) are much more comfortable with students from the same ethnic and cultural background, thus, the number of people from the same geographic region rapidly increases making the community selfselective and discouraging students from other ethnic and gender backgrounds to enter programs. Fig. 2(b) illustrates similar distribution for the four Middle Eastern universities. As clear from the chart, the diversity situation in these programs are much worse than in the US: some regions (Europe and North America) are not represented at all. While it might be a good idea to attract local talent to educate local students, these programs and, thus, their graduates would never be able to compete with those who were educated in a diverse environment, saw many different cultures and ways of solving problems.

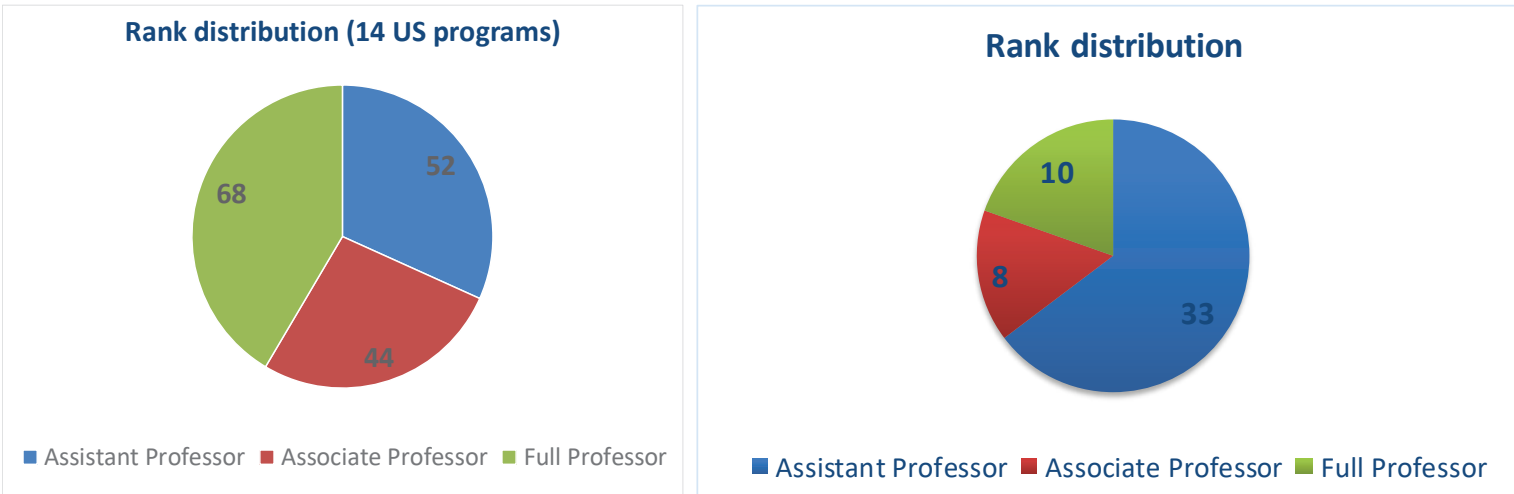


(a)

Figure 3. Faculty rank distibution in (a) the US and (b) in the Middle East based on tenure-track faculty.

Another important indication of diversity is age of university faculty. Fig. 3(a) shows rank distribution of faculty in the US for 14 chosen schools. Typically, assistant professors are relatively young faculty members from late $20 \mathrm{~s}$ to $40 \mathrm{~s}$, associated professors are from 40s to 50s and then they become full professors. As the chart shows, in the US the ratio of the three categories is almost ideal and is roughly 1:1:1 with a slight distortion toward older faculty members (full professors). Fig. 3(b) shows a completely different situation in the Middle East with about 65\% of the faculty being assistant professors. It is hard to explain why would the programs have so many junior faculty members without promoting them to associate or full rank. Either the Middle Easter universities do not retain their faculty well and, thus, have very few senior faculty members, or they have a completely different definition of academic ranks that has nothing to do with description of these ranks in the US. Perhaps, in these universities tenure is not even an option for academic staff and award of associate and full professorship has completely different criteria.

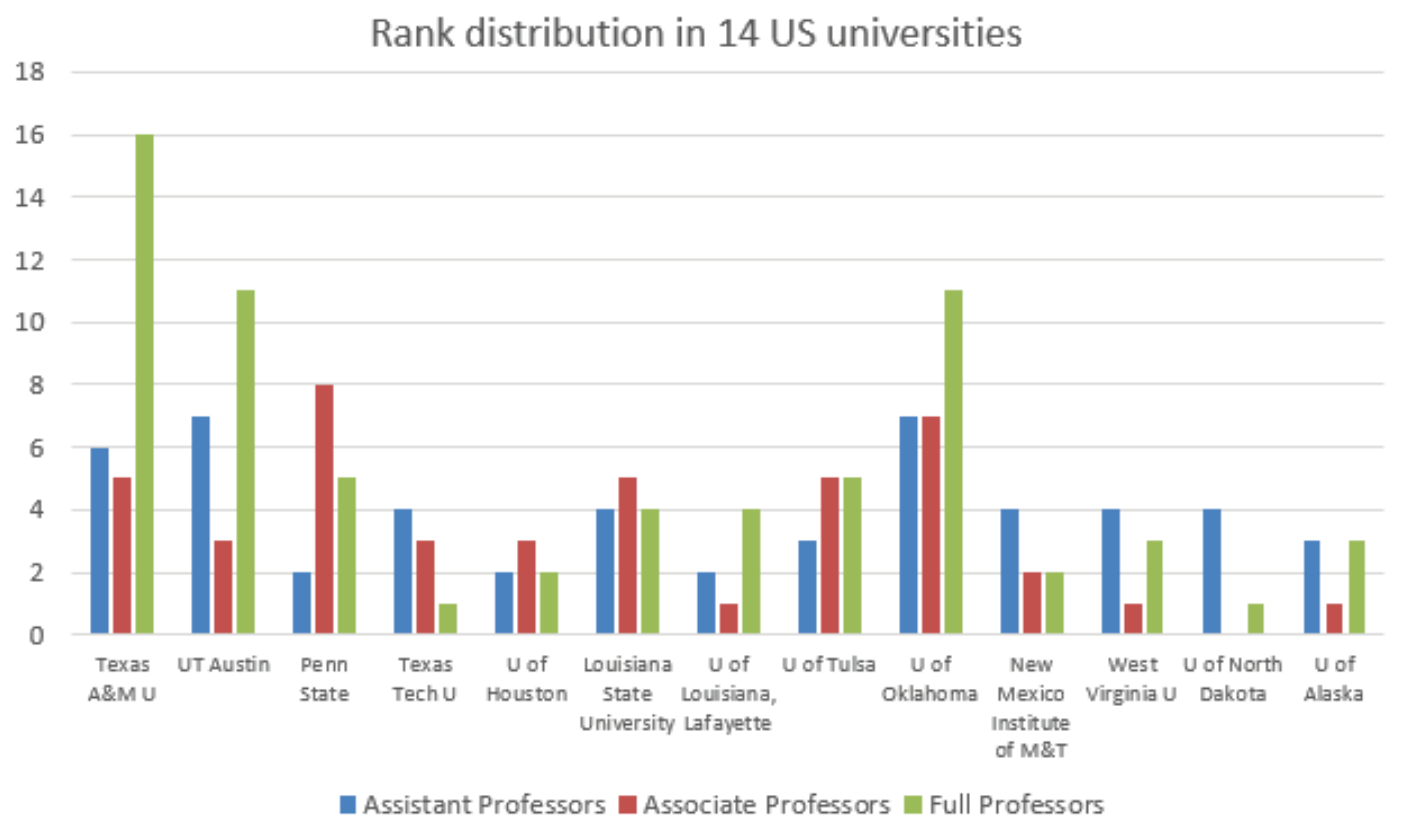

Figure 4. Faculty rank distibution in the US based on tenure-track faculty plotted by university. 
Fig. 4 provide a more detailed distribution of faculty by rank by each US university. On this plot, the reader can observe that in almost each school there is some distortion toward either rank. For example, Texas A\&M has the highest number of full professors and incomparably low numbers of associate and assistant professors. University of North Dakota, however, has no associate professor at all. These features can be explained by relative age of these programs and their school rankings. While Texas A\&M has a relatively old and well-established program that can attract by high pay many prominent scholars, University of North Dakota is a new program that cannot afford to pay for a well-known name and, thus, primarily chooses assistant professors from their own graduates.

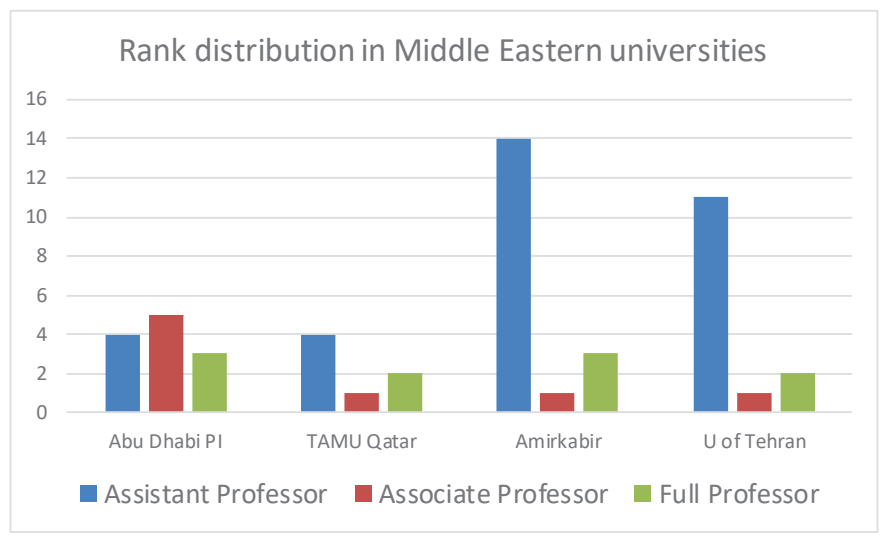

Figure 5. Faculty rank distibution in the Middle East based on tenure-track faculty plotted by university.

Fig. 5 provides similar rank distribution for the four universities in the Middle East. From this bar chart, the reader can observe that UAE and Qatar programs, being influenced by the western system of education and frequently visited by western scholars, are very similar to the majority of the US based programs. Iranian programs, on the other hand, are drastically different and more alike University of North Dakota (which is mostly staffed with the academic members of Iranian heritage). This is an interesting observation how communities with certain traits become self-selective and start looking similarly even though they are in different geographic regions. 
Faculty by Gender (14 US Programs)

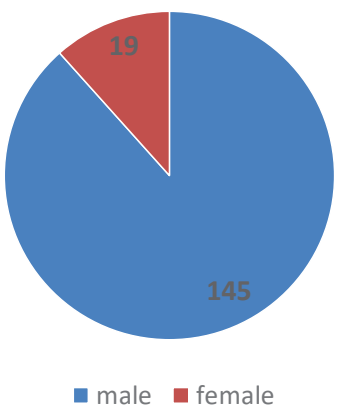

(a)

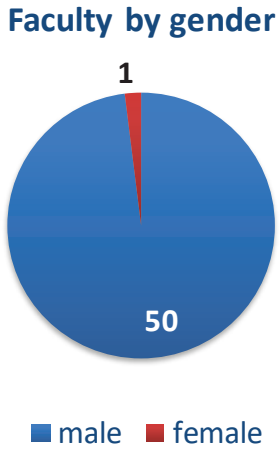

Figure 6. Faculty gender distibution in (a) the US and (b) in the Middle East based on tenure-track faculty.

The final aspect of diversity to discuss in this section is gender diversity. Petroleum engineering has been traditionally a male-dominated community. Recently, big academic programs in the US have stated their commitment to great gender diversity and made a continuous effort to attract more qualified female $\mathrm{PhD}$ graduate to their academic staff. While the ratio of men to women among $\mathrm{PhD}$ petroleum engineering students is about $3: 1$, the percent of female professors is still only $13 \%$ in the US (Fig. 6(a)). The situation with gender diversity in the Middle East is still very alarming. As shown in Fig. 6(b) in the four petroleum engineering programs selected in this study, women comprise only $2 \%$. This distortion can be easily explained by unattractiveness of these institutions (due to their regional culture) for most western female $\mathrm{PhD}$ holders and scarcity of domestic female $\mathrm{PhD}$ students.

\section{Discussion}

Demographic, ethnic, and gender diversity is key to any organization, its health and prosperity. Nonetheless, frequently we observe that, if left to their own devices, many social and professional groups become self-selective and less diverse. For example, this phenomenon can be readily observed in the academia when a professor forms a research group that is mostly comprised of people from certain origin or personal qualities. While on an individual level, this situation can be quite convenient and even beneficial, on the large scale the lack of diversity creates severe distortions and discourages many talented individuals from joining the group, organization, or industry in general. 


\section{Conclusions}

In this study, we analyzed statistical data from various petroleum engineering departments at universities from the US and the Middle East and observed some alarming trends. Our analysis revealed an unexpected picture inside the petroleum engineering discipline. For example, there is obvious inefficiency in diversity staffing in universities worldwide. We carefully investigated this phenomenon and uncovered the true reasons behind staggering demographic gaps. The statistical assessment shows a particularly alarming situation among junior faculty members coming from preferential national group which manifests in scarcity of female representatives (some schools such as University of Tulsa, Texas Tech, ULL and University of Wyoming do not have any female faculty members in their petroleum division, while the rest of the programs have barely $5-15 \%$ of the entire faculty counts with rare exceptions such as Texas A\&M and UT Austin who try to maintain greater demographic diversity). This phenomenon observed even though most programs try to keep their graduate student male to female ratio close to 3:1 with substantial number of women continuing their education toward $\mathrm{PhD}$ in recent years. Clearly, there are some discouraging factors for women to become university educators that we tried to elaborate on.

Moreover, our investigation of mobility of faculty members revealed that most schools organize conclaves of their own graduates by retaining them as faculty members which also impacts diversity in a negative way. Poor mobility leads to a rapid decline in research and demographic diversity because graduates of other schools and those with other research interests have difficulties joining these selfselective programs.

\section{References}

[1] Phillips, K. 2014. How Diversity Makes Us Smarter. Scientific American Oct. 1, 2014. Accessed at https://www.scientificamerican.com/article/how-diversity-makes-us-smarter/. 